\title{
Active Vibration Control of Rectangular Plate with Distributed Piezoelements Excited Acoustically and Mechanically
}

\author{
M. KOZUPA* AND J. WiCIAK \\ Department of Mechanics and Vibroacoustics, University of Science and Technology - AGH \\ al. Mickiewicza 30, 30-059 Kraków, Poland
}

\begin{abstract}
The paper presents simulations and research results of testing of the aluminium plate with active vibration control. The aim of this paper is to analyze and compare two ways of excitation of the test plate, various influence on its vibrations and active damping control. Vibration control of the smart structure is realized through four piezoceramic PZT actuators and one PZT sensor bonded to the plate. Simulations and numerical computations of the structure are performed in ANSYS environment. Measurements are executed on specialized sound insulation suite for small elements in reverberation chamber. At the beginning white noise sound source is used in purpose to measure basic vibration modes. After numerical computations and measurements three particular frequencies has been chosen and for them active damping is applied. There are two ways of exciting the test plate; first method is sound wave, second is mechanical vibrations via one of piezoceramics. The test results indicate that PZTs can decrease vibrations by approximately $15 \mathrm{~dB}$ for a pure sound input with acoustic excitation method, for mechanical excitation method $18 \mathrm{~dB}$ for a sinus vibration signal is achieved.
\end{abstract}

PACS numbers: 43.40.Dx, 43.50.-x

\section{Introduction}

The paper concentrates on vibration control of a plane structure using piezoceramic elements (PZT). The piezoelectric effect provides the ability to use these materials as both sensors and actuators [1]. The application of PZTs for reducing vibrations and structure-borne noise $[2-5]$ has been studied by many researchers in the past few years $[1,2,6,7]$. The passive control strategies for smart materials consist almost entirely of various shunt circuit techniques [8]. The active control is realized via two control schemes: feed forward and feedback. The vast majority of the PZT studies concentrate on the use of actively controlled PZTs for reducing vibrations and the resulting structure-borne noise. The reduction of sound radiation through vibration reduction is the goal for authors. It can generally be achieved in practice either by modifying the dynamic characteristic of the structure (passive methods) or by the application of controlled secondary source of vibration (active control). Elliott et al. [7] in particular show that the velocity distributions which radiate independently can be calculated as the eigenvectors of an elemental radiation resistance matrix which can be easily calculated for a planar radiator. These velocity distributions are termed the "radiation modes" of the surface. They obtained interesting aspect of the radiation modes, that for small values of nondimensional frequency $\Omega=k l$ their radiation efficiency falls off very rapidly with increasing mode order, where: $k=\omega / c, l$ is length, $\omega$ is the angular frequency

\footnotetext{
* corresponding author; e-mail: Michal.Kozupa@agh.edu.pl
}

of the source, $c$ is the speed of sound. The research results allow to concentrate only on the first three or four radiation modes. The shapes of the radiation modes also suggest on designing of possible distribution for sensors and actuators [11, 12].

The aim of this paper is comparison two ways of excitation: mechanical via PZT element and acoustical via loudspeaker, analyze various influence on plate vibrations. The main purpose is active damping control. In this research a simple active control based on frequency characteristic measurements and power signal applying realized in LabView system is used for control PZTs. The test plate is installed in the window between excitation and receiving chamber. White noise sound source is used to incident on a clamped at the edges thin rectangular aluminium plate in purpose to measure basic vibration modes. Two methods of exciting the test plate are used; one is mechanical via piezoceramic path, second is acoustical via loudspeaker. In both cases piezoceramics are used to damp the plate vibration. Finite element model of the smart structure and numerical computation are done in ANSYS software to obtain frequency response function and visualizations of the test plate modes.

\section{Smart structure, experimental setup}

The smart structure under study is an alluminum rectangular plate with dimensions of $453 \times 560 \times 1 \mathrm{~mm}$. Piezoceramic patches bonded to the test plate at the locations shown in Fig. 1, generates excitation by imparting a bending moment to the structure. The PZTs are acquired from Stelco GmbH, part number PZT-4D.

The size of each PZT patch is $20 \times 20 \mathrm{~mm}$ with a thickness of $1 \mathrm{~mm}$. The location of the PZTs is selected based 


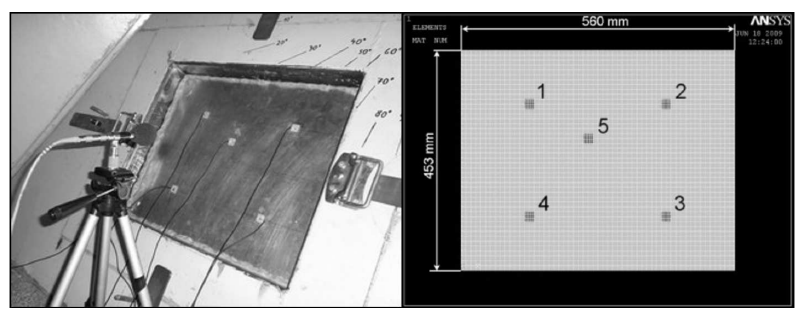

Fig. 1. Experimental setup and PZT location on plate.

on an extensive finite element and modal analysis testing. Taking into account small value of the $\Omega$ parameter, $k l=0.4$, the PZTs actuators are placed symmetrically in the centre of each quarter (Fig. 1). Controlled voltage are applied on actuators to damp the plate vibrations. Sensor position is chosen so the nodes and loops for the first sets of modes have minimum influence in order to obtain undisturbed frequency response function. The experiments are done in the set of two chambers. Source and receiving chamber are shown in Fig. 1. The source chamber is reverberant a semi - anechoic tetrahedron pyramid enclosure build inside the large reverberant chamber in its most acute corner. The test plate is installed in the opening for measuring sound reduction index for small elements. The excitation chamber enclosure is a sandwich structure made of steel and rubber with a sound insulation of $R_{w}=45 \mathrm{~dB}$ measured when the window is filled with the sample mad of the same material as the enclosure. At the edges test plate is tight with putty what gives it clamp at the edges conditions. Inside the source chamber a loudspeaker is placed. The active control system is made in LabView environment and executed by Multifunction Data Acquisition system NI PCI-6230.

\section{Numerical model and simulations}

The test plate is modelled in ANSYS software. Aluminium plate is represented using finite element of SOLID 45 type for the 3-D modelling of solid structures with three degrees of freedom: UX, UY, UZ and PZT using elements of SOLID 5 type with four degrees of freedom: UX, UY, UZ and VOLT. The test plate is meshed with $5 \times 5 \times 0.25 \mathrm{~mm}$ elements and PZTs with $1.25 \times 1.25 \times 0.25 \mathrm{~mm}$ elements. All the material properties of piezoceramic are build in including permittivity, piezoelectric stress and elastic stiffness matrix. Boundary conditions are performed as if the test plate is clamped at the edges.

TABLE I

Natural frequencies for theoretical calculation and FEM model.

\begin{tabular}{c|c|c|c|c|c}
\hline \hline 0 & 1 & 2 & 3 & 4 & 5 \\
\hline Mode & Theoretically & ANSYS & Difference & ANSYS PZT & Difference \\
\hline 1.1 & 36.7 & 37.0 & $0.4 \%$ & 36.7 & $0.8 \%$ \\
1.2 & 65.2 & 65.2 & $-0.3 \%$ & 64.6 & $1.0 \%$ \\
2.1 & 83.9 & 84.7 & $0.7 \%$ & 83.7 & $1.2 \%$ \\
2.2 & 109.8 & 110.7 & $0.5 \%$ & 108.1 & $2.3 \%$ \\
1.3 & 111.2 & 111.0 & $-0.4 \%$ & 110.0 & $0.9 \%$ \\
2.3 & 153.4 & 154.4 & $0.2 \%$ & 151.3 & $2.0 \%$ \\
3.1 & 155.7 & 157.6 & $0.9 \%$ & 156.6 & $0.7 \%$ \\
1.4 & 173.5 & 173.3 & $-0.4 \%$ & 173.5 & $-0.1 \%$ \\
3.2 & 180.6 & 182.7 & $0.7 \%$ & 179.9 & $1.5 \%$ \\
2.4 & 214.3 & 215.2 & $0.0 \%$ & 215.1 & $0.1 \%$ \\
3.3 & 222.5 & 224.9 & $0.6 \%$ & 221.9 & $1.3 \%$
\end{tabular}

The analysis is made for the first 11 modes. Block Lanczos method is used for mode extraction. It uses the algorithm where the Lanczos recursion is performed with a block of vectors. The basic equation in mode extraction is the classical eigenvalue problem:

$$
[K]\left\{\phi_{i}\right\}=\omega_{i}^{2}[M]\left\{\phi_{i}\right\},
$$

where: $[K]$ is stiffness matrix, $\left\{\phi_{i}\right\}$ is mode shape vector, $\omega_{i}$ is frequency of mode and $[M]$ is mass matrix. Resonant frequencies and the difference between the theoretical model and FEM model in Ansys are shown in Table I. Column 1 contains modal frequencies for clamped plate calculated with approximation methods outlined by Warburton [13]. Column 2 and 4 contain respectively modal frequencies for clamped at the edges aluminium plate without and with PZT patches. Column 3 and 5 summa- 
rizes respectively percentage difference of column 1 and 2 as well as 2 and 4 .

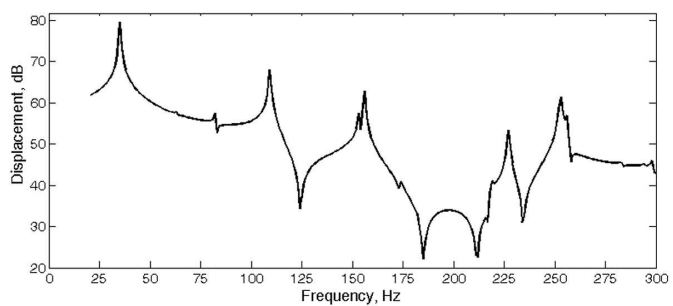

Fig. 2. Frequency response function of test plate Ansys model.

Results for harmonic analysis of the FEM model is shown in Fig. 2. Displacement is calculated for node located on PZT (5) sensor with reference level $10^{-12} \mathrm{~m}$. The structure's response is computed over the frequency bandwidth, 30-300 Hz, with a $1 \mathrm{~Hz}$ increment.
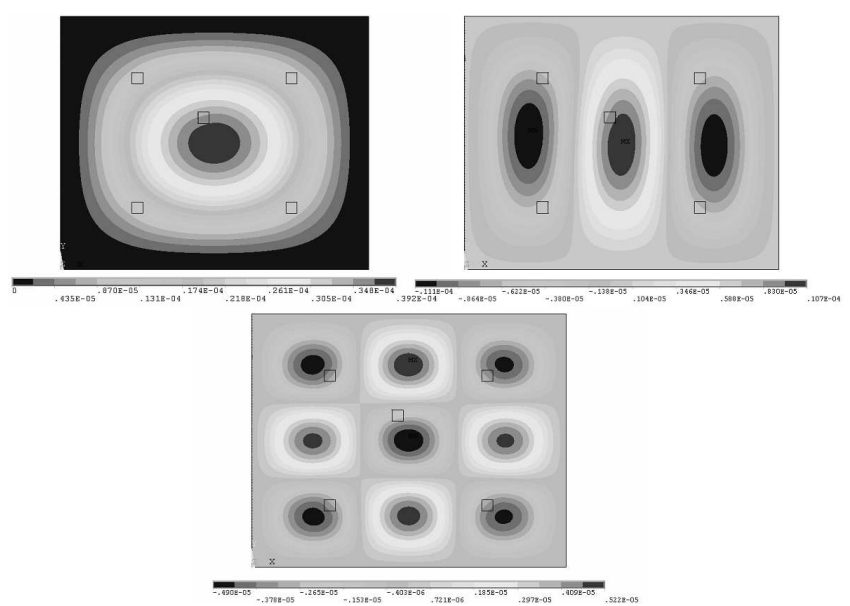

Fig. 3. Mode shapes for chosen modes.

\section{Results and discussion}

The measurement proceeding is consecutively frequency characteristic measurements of vibration (Fig. 4), subsequently choosing frequencies of mode vibration in which active control is implemented. The characteristic is obtained by exciting the test plate with acoustic white noise and registered response signal from all five PZT elements. Frequency characteristic of vibration (Fig. 4) allow to localize basic modes, collaboratively with FEM model, free vibration form. It can be seen that mode (1.1) match to $39 \mathrm{~Hz}$, mode (1.3) to $100 \mathrm{~Hz}$ and mode (3.3) correspond to $190 \mathrm{~Hz}$. The smart structure is excited to vibrate in two ways: pure sound (speaker) and mechanically $(\mathrm{PZT})$. In parallel controlling signal is applied for damping vibration. In both cases the exciting signal is pure tone sinus at specific frequency.

Changes of extracted modes frequencies calculated in ANSYS Software compared to frequency characteristic

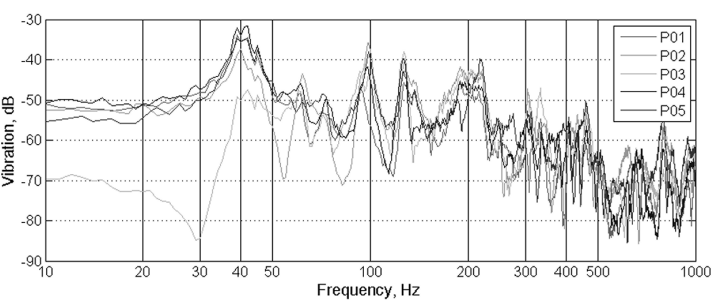

Fig. 4. Frequency response function for plate vibrations.

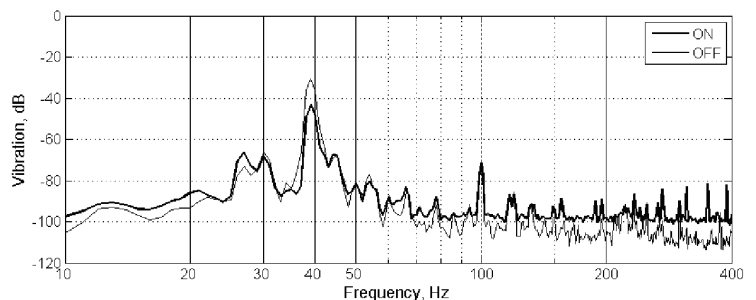

Fig. 5. Frequency characteristic for plate vibration reduction at $39 \mathrm{~Hz}$, acoustic.

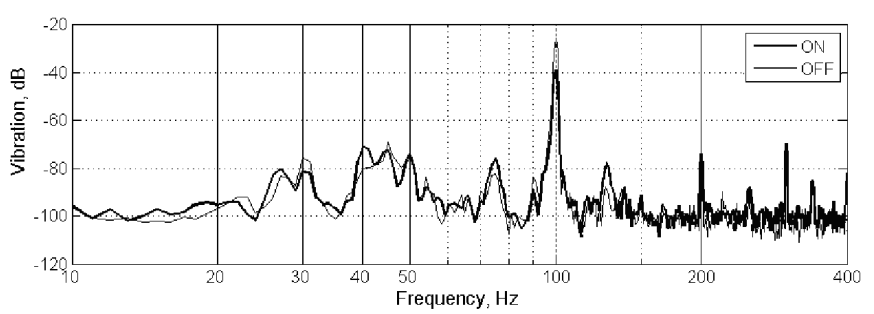

Fig. 6. Frequency characteristic for plate vibration reduction at $100 \mathrm{~Hz}$, acoustic.

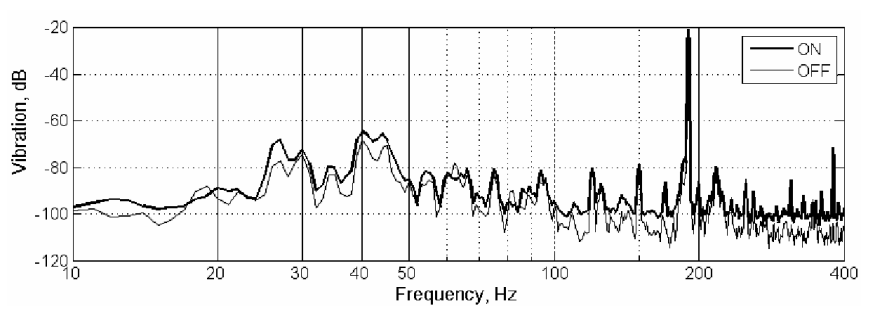

Fig. 7. Frequency characteristic for plate vibration reduction at $190 \mathrm{~Hz}$, acoustic.

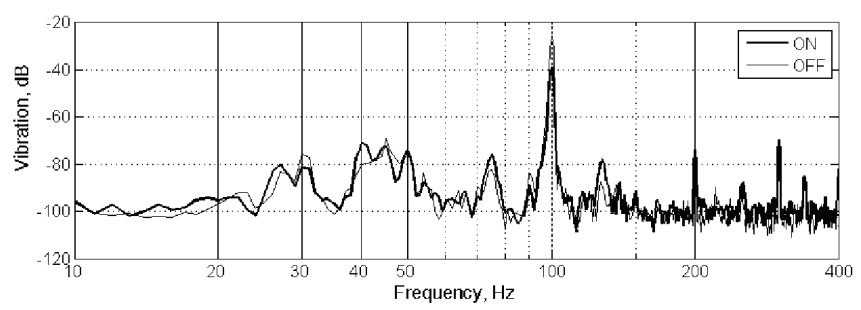

Fig. 8. Frequency characteristic for plate vibration reduction at $100 \mathrm{~Hz}$, mechanical. 


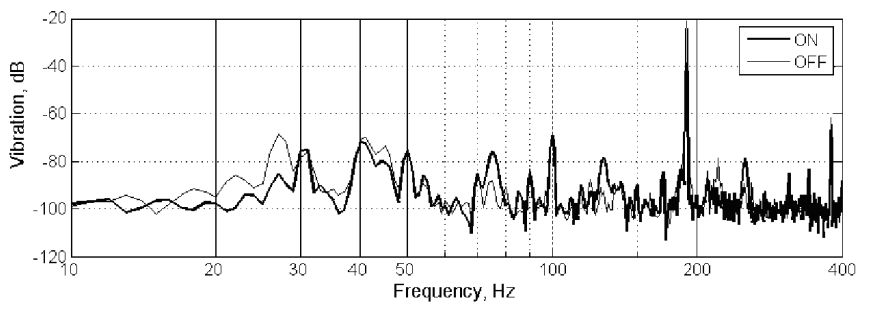

Fig. 9. Frequency characteristic for plate vibration reduction at $190 \mathrm{~Hz}$, mechanical.

obtained in measurement are probably caused by imperfection of boundary conditions in experiment and other methods of excitation the test plate. Figure 3 shows visual representation of modes which are chosen to reduce its vibration. Mode (1.1) appears at about $37 \mathrm{~Hz}$, mode (1.3) at about $110 \mathrm{~Hz}$ and mode (3.3) at about $222 \mathrm{~Hz}$. Comparison with obtained by the measurement frequencies at experimental setup which are accordingly $39 \mathrm{~Hz}$, $100 \mathrm{~Hz}$ and $190 \mathrm{~Hz}$ gives acceptable results.

Figures 5-9 shows the vibrations plotted versus frequency for the uncontrolled plate - blue bars and the controlled plate - red line, using the two excitation approaches described earlier. Damping signal is sinus signal changed in phase in comparison with excitation signal. Frequency characteristic of vibration for pure sound excitation is shown in Figs. 5-7, in turn frequency characteristic for mechanical excitation presents Figs. 8, 9. The largest level of attenuation is obtained for mode (3.3) around $190 \mathrm{~Hz}$. Very good attenuation is also seen for the other modes present in the frequency bandwidth except for the resonance at $190 \mathrm{~Hz}$ for acoustic excitation of the plate. When the excitation method is loudspeaker it can be observed increase of vibrations at noise level above $100 \mathrm{~Hz}$. In contrary the RMS level for the whole spectrum decreases. Table I correlate data in regard to measured and calculated results.

\section{TABLE II}

Vibrations decrease in resonance frequencies of testing plate.

\begin{tabular}{c|c|c|c|c|c|c}
\hline \hline freqency [Hz] & \multicolumn{2}{|c|}{39} & \multicolumn{2}{c|}{100} & \multicolumn{2}{c}{190} \\
\hline force & acou. & mech. & acou. & mech. & acou. & mech. \\
\hline tone & -12 & - & -15 & -14 & $\mathbf{2 6}$ & -18 \\
RMS & -12 & - & -14 & -13 & $\mathbf{2 6}$ & -18
\end{tabular}

We could not manage to excite the test plate at $39 \mathrm{~Hz}$ with PZT in regard of to small dimensions of piezoceramic and to low level of control signal, possible reason can also be location of PZT patches.

\section{Conclusions}

The aim of experiment is damping the plate vibrations, obtained results are encouraged. Two ways of excitation of the test plate are applied.
First method is sound wave and second one mechanical vibrations via one of piezoceramic. The simplest one sensor active control successfully reduced the vibration level at the first three resonance frequencies by $12-18 \mathrm{~dB}$.

Considering the interaction between the vibrating structure and the acoustic field, the effort on vibration reduction can be applied in sound radiation reduction. It is known that, the greater the mode number, the less efficient sound radiation is. That is why controlling modes considered in the paper should give satisfactory results.

Some initial researches has already been done. Because of some limitations the test plate is going to be replaced by bigger one, with dimensions of $0.5 \mathrm{~m}^{2}$. Nine PZT patches will be used for active control.

The results of this study confirm the previously observed benefits of the PZTs for reducing vibrations to increasing transmission loss in structures.

\section{Acknowledgments}

This research work is supported by the Polish Ministry of Science and Higher Education grant no. NN504282737.

\section{References}

[1] M. Ahmadian, K.M. Jeric, J. of Sound and Vibration 347, (2001).

[2] A. Brański, S. Szela, On the quasi optimal distribution of PZTs in active reduction of the triangular plate vibration, Archives of Control Sciences 17, 4, 357 (2007).

[3] M.S. Kozień, J. Wiciak, Archives of Acoustics 33, 643 (2008).

[4] M.S. Kozień, J. Wiciak, Acta Phys. Pol. A 116, 348.

[5] M. Kozupa, Proc. 56th Open Seminar Acoustics, Institute of Fundamental Technological Research, Committee on Acoustics, Polish Acoustical Society, 2009, p. 347 .

[6] Bor-Tsuen Wang, C.R. Fuller, K. Dimitriadis Emilios, The J. Acoustical Society of America, 2820 (1991).

[7] S.J. Elliott, M.E. Johnson, J. Acoustical Soc. America, 2194 (1993).

[8] G.A. Lesieutre, Shock Vibration Digest 187, (1998).

[9] W.P. Rdzanek, Jr., W.J. Rdzanek, Z. Engel, J. Sound Vibr. 265, 155 (2003).

[10] W.P. Rdzanek Jr., W.J. Rdzanek, K. Szemela, Archives of Acoustics 34, 75 (2009).

[11] J. Wiciak, Pol. J. Environmental Studies 13 Supplement III, 125 (2004).

[12] B. Xiaoqi, V.V. Varadan, V.K. Varadan, Smart Materials and Structures 4, 231 (1995).

[13] G.B. Warburton, Proceedings of the Institute of Mechanical Engineers, (1954). 